\title{
O K-CPT em uma amostra brasileira: descrição do desempenho e comparação com as normas norte-americanas
}

\author{
K-CPT in a Brazilian sample: description of performance and comparison \\ with North American norms
}

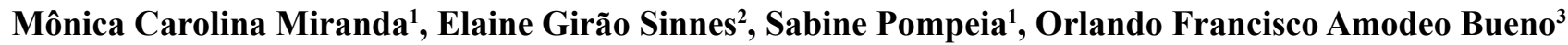 \\ ${ }^{1}$ Doutora, pesquisadora. ${ }^{2}$ Especialista, psicóloga. ${ }^{3}$ Livre-docente, professor adjunto, Universidade Federal de São Paulo (UNIFESP), São Paulo, SP.
}

\section{Resumo}

Introdução: O presente estudo investigou o desempenho de uma amostra de crianças brasileiras de 4 a 5 anos de idade no Conners' Kiddie CPT (K-CPT), uma tarefa computadorizada utilizada na avaliação da atenção e do controle inibitório em crianças.

Método: Os escores de crianças brasileiras, que passaram por uma triagem para excluir déficit de atenção, foram comparados com as normas norte-americanas.

Resultados: Os efeitos de gênero e idade na nossa amostra foram similares aos descritos na literatura. Entretanto, a falta de triagem para déficit de atenção pode ter distorcido os dados normativos norte-americanos, pois meninos e meninas brasileiros tiveram melhores escores em quase todas as medidas.

Conclusões: Os procedimentos de triagem aqui empregados, juntamente com a obtenção dos efeitos de idade e gênero previamente descritos, confirmam a adequação dos escores obtidos como referência local de desempenho, que podem ser úteis na avaliação de crianças pré-escolares no Brasil até que normas nacionais sejam estabelecidas.

Descritores: Teste de desempenho contínuo, crianças pré-escolares, atenção, estudo comparativo.

\begin{abstract}
Introduction: This study investigated the performance of a sample of Brazilian children aged 4-5 years on the Conners' Kiddie CPT (K-CPT), a computer-based task used to assess attention and inhibitory control in children.

Methods: Scores of Brazilian children pre-screened for attention disorders were obtained using the K-CPT, and data were compared with North American norms.

Results: Age and gender effects on the Brazilian sample were similar to those previously described. However, the lack of screening for attention deficits might have distorted normative scores of the K-CPT in the USA because Brazilian boys and girls had better scores than the North American children in almost all measures.

Conclusion: The screening procedures used in Brazil, in addition to the previously described age and gender effects on sustained attention, indicate that the results described in the present study constitute appropriate local performance scores and, as such, are useful in the evaluation of pre-school children in Brazil until national norms are established.

Keywords: Continuous performance test, preschool children, attention, cross-cultural comparative study.
\end{abstract}

\section{Correspondência:}

Mônica Carolina Miranda, Departamento de Psicobiologia, Universidade Federal de São Paulo (UNIFESP), Rua Embaú, 54, CEP 04039-060, São Paulo, SP. Tel.: (11) 5549.6899, (11) 5081.5496, Fax: (11) 5572. 5092. E-mail: mirandambr@yahoo.com.br

Fundação de Amparo à Pesquisa do Estado de São Paulo (FAPESP) (02/09395-3) e Associação Fundo de Incentivo à Psicofarmacologia (AFIP).

Não há conflitos de interesse associados à publicação deste artigo.

Copyright (C) Revista de Psiquiatria do Rio Grande do Sul - APRS 


\section{Introdução}

Os testes de desempenho contínuo (do inglês Continuous Performance Test - CPT) têm se tornado cada vez mais populares como uma maneira objetiva de medir aspectos relacionados à atenção ${ }^{1} \mathrm{e}$ frequentemente são utilizados para auxiliar no diagnóstico de distúrbios de atenção, tais como o transtorno do déficit de atenção e hiperatividade (TDAH). No momento, a versão comercial mais popular de um CPT provavelmente é o Conners' Continuous Performance Test (CPT II $\left.{ }^{2}\right)$, uma tarefa visual computadorizada para avaliação da atenção sustentada. O Conners' CPT-II pode ser aplicado em crianças a partir dos 6 anos de idade e é amplamente utilizado na investigação de transtornos do déficit de atenção em crianças em idade escolar ${ }^{3-5}$.

Estudos recentes têm demonstrado que os sintomas de TDAH são bastante comuns em crianças menores de 6 anos $^{6}$. Um terço de todos os comportamentos de TDAH, listados em questionários baseados no DSM-IV, estão presentes em, pelo menos, $40 \%$ de todas as crianças ${ }^{6}$. Contudo, o desenvolvimento de instrumentos de diagnóstico direcionados à avaliação neuropsicológica de crianças em idade pré-escolar parece estar significativamente defasado em relação aos instrumentos disponíveis para as crianças em idade escolar ${ }^{7,8}$. Uma exceção é o Conners' Kiddie CPT ${ }^{9}$ (K-CPT), que foi desenvolvido recentemente para avaliar distúrbios de atenção nessa faixa etária e que também pode ser utilizado para avaliação da eficácia de tratamento nessa população .

Comparado aos CPTs anteriores, o K-CPT é mais curto (7,5 minutos em vez de 14 minutos) e utiliza figuras em vez de letras como estímulos. O intervalo interestímulo (IIE) é de 1,5 ou 3 segundos, e os estímulos são apresentados por 500 milissegundos.

Os dados normativos dos EUA, que são descritos no manual do teste ${ }^{9}$, são baseados em uma amostra não-clínica de 314 crianças norte-americanas, com idades entre 4 e 5 anos. Os autores analisaram os tempos de reação e os erros de omissão e coomissão e descobriram que os escores melhoraram com a idade, em concordância com o observado em crianças mais velhas $^{10} \mathrm{e}$ em crianças entre 3 e 6 anos através da utilização de um paradigma diferente $\left(\mathrm{C}-\mathrm{CPT}^{8}\right)$. Não foram relatados efeitos de gênero. Contudo, em crianças mais velhas (com idades entre 6 e 8 anos) ${ }^{10}$, é observado que as meninas apresentam menos erros de coomissão, melhor capacidade de detecção de sinais e são mais cautelosas $(\beta)$ ao reagir ao teste, mas cometem mais erros de omissão e têm tempos de reação mais longos e menos consistentes do que os meninos ${ }^{5,10}$. No único estudo que avaliou as diferenças entre gêneros no desempenho de crianças mais novas (de 3 a 6 anos), não foram encontrados efeitos de gênero através do uso do C-CPT ${ }^{8}$.

Existem poucos estudos transculturais comparando as normas de diferentes países através do uso do Conners' $\mathrm{CPT}$, e nenhum dos que tem sido publicado utilizou o K-CPT. Porém, a necessidade de normas locais é amplamente reconhecida, já que fatores culturais podem influenciar o desempenho cognitivo $^{11}$. Em nosso estudo com uma amostra brasileira de crianças em idade escolar utilizando o Conners' $\mathrm{CPT} \mathrm{II}^{5}$, os efeitos de idade e gênero são similares àqueles encontrados em outros estudos com o Conners' CPT II. Contudo, ao comparar as amostras brasileira e norte-americana, os resultados mostraram que as crianças brasileiras tiveram escores melhores na maioria das medidas. Miranda et al..$^{5}$ concluíram que esses resultados conflitantes podem ser devido a diferenças na seleção da amostra, com os resultados norte-americanos sendo determinados sem pré-triagem para possíveis déficits de atenção ou distúrbios do desenvolvimento. Portanto, as normas norte-americanas podem ter sido estabelecidas considerando a inclusão de crianças com possíveis problemas de atenção, o que pode ter reduzido os escores globais de desempenho. Isso é particularmente preocupante, já que as normas dessa tarefa são utilizadas com o objetivo de estabelecer um diagnóstico ${ }^{4,12}$. Considerados em conjunto, esses achados sugerem que dados locais devem ser obtidos em diferentes culturas para serem usados como escores locais de referência. Também é importante estabelecer normas para o auxílio no diagnóstico de distúrbios de atenção não contaminadas por dados de sujeitos que podem ter o distúrbio avaliado pelo instrumento.

Assim, o objetivo do presente estudo foi o de determinar o desempenho no K-CPT em uma amostra de crianças brasileiras com idades entre 4 e 5 anos. Foram realizadas análises de efeitos de idade e gênero, e os dados foram comparados com os dados das normas norte-americanas padronizadas?

\section{Método}

As crianças selecionadas como sujeitos para o presente estudo eram estudantes de escolas públicas e particulares da cidade de São Paulo e representavam a distribuição de classes sociais dessa cidade informada pela Associação Brasileira de Institutos de Pesquisa de Mercado (ABIPEME).

As crianças selecionadas inicialmente eram os números impares da lista de chamada das escolas e estavam matriculadas em séries adequadas para suas idades, totalizando 312 crianças com idades entre 4 e 5 anos. O segundo passo consistiu na aplicação da escala abreviada de Conners adaptada para a população brasileira ${ }^{13}$, uma ferramenta de triagem amplamente utilizada no processo de seleção em pesquisas sobre pacientes com TDAH ${ }^{13}$. A pontuação da escala é determinada pelo professor das crianças e avalia problemas de comportamento, tais como hiperatividade e desatenção. As 10 crianças que apresentaram escores acima do ponto de corte para idade e gênero, segundo as normas brasileiras da escala de Conners ${ }^{13}$, foram excluídas.

A seguir, os pais foram convidados a assinar os formulários de consentimento informado que autorizam a participação das crianças. Os pais de 82 crianças não responderam ou não autorizaram a participação de seus filhos. Dezessete crianças foram excluídas por apresentarem um histórico que poderia estar relacionado a distúrbios de desenvolvimento (meningite, trauma encefálico, convulsão e dificuldades de aprendizagem). 
Após essas exclusões, um total de 104 crianças realizou o teste K-CPT. Dez testes foram invalidados por falha na execução da tarefa ou porque os dados não foram registrados pelo programa, e três crianças foram excluídas por serem casos atípicos. A amostra final foi composta por 91 crianças.

\section{Procedimentos}

Todos os procedimentos do presente estudo foram aprovados pelo Comitê de Ética e Pesquisa - UNIFESP. O Conners' K-CPT foi realizado exatamente de acordo com a descrição do manual ${ }^{9}$. As crianças foram avaliadas individualmente em suas escolas, em salas com iluminação apropriada e baixos níveis de ruído. O teste foi apresentado em um laptop. O tempo total para a aplicação foi de aproximadamente 7,5 minutos após um treinamento inicial que garantiu a compreensão das instruções pelas crianças.

Durante a tarefa, as figuras são apresentadas na tela e a criança é instruída a pressionar a tecla de espaço sempre que uma figura aparecer, exceto se a figura for uma bola. As figuras são mostradas durante 500 milissegundos. Existem cinco blocos, com dois sub-blocos cada de 20 tarefas, um com 1,5 segundos de IIE e outro com 3 segundos de IIE.

As seguintes medidas geradas pelo programa de teste foram analisadas.

1. Erros: mede a falta de exatidão da resposta, sendo dividida em duas categorias:

a) Omissão: número de alvos aos quais o sujeito não consegue responder, ou seja, quando a resposta não é dada (sendo omitida) para figuras que não são a bola. Taxas altas normalmente indicam respostas não-orientadas e lentas.

b) Coomissão: número de vezes que o sujeito responde a um estímulo que não é o alvo, ou seja, quando a respostas é dada à figura da bola. Um tempo de reação (TR) longo combinado com um grande número de erros de omissão e coomissão indica desatenção, enquanto que um TR rápido combinado com muitos erros de coomissão, mas poucos erros de omissão, reflete impulsividade.

2. Tempo de Reação (TR): velocidade e consistência da resposta medidos em milissegundos e que são maiores do que 100 milissegundos (ver perseverações abaixo). Os seguintes subtipos de medidas são fornecidos:

a) TR da Batida: TR médio para todas as respostas-alvo em todos os tempos dos blocos.

b) Erro Padrão do TR (EP-TR): consistência do TR expresso como EP para as respostas ao alvo. Escores altos indicam respostas altamente variáveis, geralmente relacionadas a desatenção.

c) Variabilidade do EP: variabilidade apresentada pelos sujeitos ao seu próprio EP geral.

3. Medidas derivadas da Teoria de Detecção de Sinais:

a) Detectabilidade (d'): uma medida do nível de discriminação dos sujeitos entre alvos e não-alvos. Valores de d' mais altos indicam melhor discriminação entre o alvo e os estímulos distratores.

b) Estilo da Resposta ( $\beta$ ): indica o critério de resposta dos sujeitos. Sujeitos cautelosos, que não respondem com frequência, apresentam valores mais altos neste índice. Os sujeitos que respondem mais livremente e estão menos preocupados com a possibilidade de falha têm taxas mais baixas.

c) Perseverações: devido às limitações fisiológicas, não é possível responder em menos de 100 milissegundos após os estímulos serem apresentados; portanto, os tempos de respostas mais rápidos do que 100 milissegundos são classificados como perseverações. Uma taxa alta de perseverações é resultado de respostas antecipatórias (indicando impulsividade), respostas aleatórias (indicando grave prejuízo), ou respostas muito lentas aos estímulos anteriores (indicando desatenção).

4. Resultados por Blocos: o K-CPT é apresentado em cinco blocos, o que permite a avaliação de mudanças no tempo, na vigilância e também na consistência das respostas com a progressão do teste. Duas medidas são fornecidas:

a) Mudanças de TR por Bloco: mudanças do TR médio dos blocos no decorrer dos blocos. Valores positivos indicam um TR que vai se tornando lento à medida que os blocos são apresentados, enquanto que valores negativos indicam que o TR é mais rápido à medida que o teste progride.

b) EP das mudanças de TR por bloco: mudanças nos EP do TR à medida que os blocos são apresentados. Valores positivos indicam menos consistência nos tempos de respostas, sugerindo uma possível perda da vigilância, enquanto que valores negativos indicam maior consistência de reação à medida que o teste progride.

5. Resultados por IIE: possibilitam a avaliação da capacidade dos sujeitos de se ajustarem a IIE diferentes. Há duas medidas:

a) Mudanças de TR por IIE: mudanças no TR médio nos sub-blocos com diferentes IIE. Valores positivos indicam um TR mais lento à medida que o IIE aumenta, enquanto que valores negativos indicam um TR mais rápido.

b) EP das mudanças de TR por IIE: mudanças no EP médio nos sub-blocos com diferentes IIE. Valores positivos indicam menos consistência no TR durante IIE mais longos, e valores negativos indicam mais consistência durante IIE mais longos.

\section{Análise estatística}

Os programas de estatística utilizados foram o SPSS versão 11.0 e o SAS's proc. GLM versão 8.01. O nível de significância adotado foi um $\mathrm{p}<0,05$.

O desempenho da amostra de crianças do presente estudo, de acordo com a idade (em anos) e gênero, foi determinado 
através dos escores de cada variável disponibilizados pelo pacote do programa, exceto para o escore $\mathrm{T}$ e os percentis, porque essas variáveis são valores transformados em relação aos dados normativos obtidos nos EUA. Para avaliar os efeitos de idade e gênero, foram aplicados testes de ANOVA bidirecionais para cada uma das variáveis individuais. Para a análise de proporção das crianças em cada gênero por faixa etária (4 e 5 anos), o teste de qui-quadrado foi utilizado. Para a análise da idade média de cada gênero nas duas faixas etárias, o teste $t$ de Student para amostras independentes foi realizado. A comparação entre os dados obtidos dos autores brasileiros e os dados fornecidos pelos autores da padronização norte-americana foi realizada por faixa etária e gênero, utilizando-se o teste $Z$ para uma média com variância conhecida ${ }^{14}$. A comparação entre as amostras brasileira e norte-americana em relação à idade média em cada faixa etária, ao número de meninos e meninas e ao status socioeconômico não foi realizada devido ao fato de tais dados das normas norte-americanas não terem sido fornecidos pelos autores.

\section{Resultados}

\section{Amostra brasileira}

A Tabela 1 mostra a distribuição da amostra do presente estudo em relação à faixa etária e ao gênero; a Tabela 2 mostra os resultados estatísticos; e a Tabela 3 apresenta os escores: o número de sujeitos dos gêneros masculino e feminino foi similar em todas as faixas etárias $(p=0,75)$, e a idade média de meninos e meninas nas duas faixas etárias foi equivalente $(\mathrm{p}=0,29)$. Não houve interação entre os efeitos de gênero e de idade. Os efeitos de gênero revelaram que as crianças de 4 anos tiveram piores escores nas medidas de porcentagem de omissões, TR, EP do TR, variabilidade,
Tabela 1 - Número de crianças da amostra brasileira por idade e gênero

\begin{tabular}{|c|c|c|}
\hline Faixa etária/Gênero & $\begin{array}{c}\text { Idade em anos } \\
\text { Média (DP) }\end{array}$ & $\mathbf{n}$ \\
\hline \multicolumn{3}{|l|}{4} \\
\hline Feminino & $4,6(0,2)$ & 23 \\
\hline Masculino & $4,5(0,2)$ & 19 \\
\hline \multicolumn{3}{|l|}{5} \\
\hline Feminino & $5,4(0,3)$ & 21 \\
\hline Masculino & $5,5(0,3)$ & 28 \\
\hline
\end{tabular}

$\mathrm{DP}=$ desvio padrão.

perseverações, mudanças de TR por IIE e EP das mudanças de TR por IIE. Os efeitos de gênero mostraram que as meninas tiveram porcentagem mais baixa de erros de coomissão e índices mais altos de d' e $\beta$.

\section{Comparação das amostras brasileira e norte-ameri- cana (Tabelas 4 e 5)}

Tanto os meninos quanto as meninas de 4 anos da amostra brasileira apresentaram melhores escores na porcentagem de erros de omissão, no TR, no EP do TR e nas perseverações. A medida de EP das mudanças de TR por bloco foi significativamente melhor somente para os meninos brasileiros de 4 anos, enquanto que as meninas brasileiras tiveram um desempenho melhor na medida de mudanças de TR por IIE. As demais medidas para as crianças de 4 anos nas duas amostras não apresentaram diferença significativa. Melhores escores também foram observados nos meninos e nas meninas brasileiros de 5 anos para porcentagem de

Tabela 2 - Valores de F e p do teste de ANOVA considerando gênero, idade e efeitos de interação na amostra brasileira

\begin{tabular}{lcccccc}
\hline & \multicolumn{2}{c}{ Gênero } & \multicolumn{2}{c}{ Idade } & \multicolumn{2}{c}{ Gênero x idade } \\
\cline { 2 - 7 } Variáveis & $\mathbf{F}$ & $\mathbf{p}$ & $\mathbf{F}$ & $\mathbf{p}$ & $\mathbf{F}$ & $\mathbf{p}$ \\
\hline \% de omissões & 0,81 & 0,37 & 19,12 & $<0,0001$ & 0,76 & 0,38 \\
\% de coomissões & 6,89 & 0,01 & 0,82 & 0,37 & 1,16 & 0,29 \\
TR (ms) & 0,12 & 0,73 & 10,84 & 0,002 & 3,32 & 0,07 \\
EP do TR & 0,80 & 0,37 & 17,71 & $<0,0001$ & 2,50 & 0,12 \\
Variabilidade & 1,07 & 0,30 & 16,08 & 0,0001 & 1,56 & 0,22 \\
Detectabilidade (d') & 10,39 & 0,002 & 1,22 & 0,27 & 0,13 & 0,72 \\
Estilo de resposta ( $\beta)$ & 4,08 & 0,05 & 1,69 & 0,20 & 0,63 & 0,43 \\
Perseverações & 1,64 & 0,20 & 4,36 & 0,04 & 0,51 & 0,48 \\
Mudanças de TR por bloco & 1,35 & 0,25 & 2,92 & 0,09 & 2,84 & 0,10 \\
EP das mudanças de TR por bloco & 0,97 & 0,33 & 0,29 & 0,60 & 5,49 & 0,06 \\
Mudanças de TR por IIE & 2,29 & 0,14 & 7,76 & 0,007 & 1,74 & 0,19 \\
EP das mudanças de TR por IIE & 0,82 & 0,37 & 7,71 & 0,007 & 2,05 & 0,16 \\
\hline
\end{tabular}

$\mathrm{EP}=$ erro padrão; IIE = intervalo interestímulo; $\mathrm{ms}=$ milissegundos; $\mathrm{TR}=$ tempo de reação. 
Tabela 3 - Média e desvio padrão em idade e em cada medida do CPT da amostra brasileira

\begin{tabular}{|c|c|c|c|c|c|c|c|c|}
\hline \multirow[b]{3}{*}{ Variáveis } & \multicolumn{4}{|c|}{4 anos } & \multicolumn{4}{|c|}{5 anos } \\
\hline & \multicolumn{2}{|c|}{$\begin{array}{c}\text { Meninos } \\
(n=19)\end{array}$} & \multicolumn{2}{|c|}{$\begin{array}{c}\text { Meninas } \\
(n=23)\end{array}$} & \multicolumn{2}{|c|}{$\begin{array}{l}\text { Meninos } \\
(n=28)\end{array}$} & \multicolumn{2}{|c|}{$\begin{array}{c}\text { Meninas } \\
(n=21)\end{array}$} \\
\hline & Média & DP & Média & DP & Média & DP & Média & DP \\
\hline$\%$ de omissões & 10,98 & 5,69 & 13,59 & 10,05 & 5,83 & 5,72 & 5,88 & 5,05 \\
\hline$\%$ de coomissões & 48,53 & 22,52 & 32,61 & 18,50 & 40,00 & 18,72 & 33,33 & 22,13 \\
\hline $\mathrm{TR}$ & 771,31 & 156,8 & 728,5 & 170,3 & 685,9 & 125,9 & 622,8 & 93,3 \\
\hline EP do TR & 33,03 & 13,97 & 26,93 & 12,99 & 18,79 & 8,21 & 20,48 & 11,54 \\
\hline Variabilidade & 38,13 & 18,58 & 30,91 & 14,46 & 22,17 & 13,24 & 21,5 & 13,73 \\
\hline Detectabilidade (d) & 0,55 & 0,38 & 0,85 & 0,36 & 0,68 & 0,33 & 0,91 & 0,50 \\
\hline Estilo de resposta $(\beta)$ & 1,83 & 1,44 & 3,46 & 3,63 & 1,54 & 1,37 & 2,25 & 3,67 \\
\hline Perseverações & 3,68 & 4,20 & 2,43 & 2,84 & 1,93 & 2,73 & 1,57 & 1,80 \\
\hline Mudanças de TR por bloco & $-0,05$ & 0,16 & 0,00 & 0,05 & 0,01 & 0,04 & 0,00 & 0,05 \\
\hline EP das mudanças de TR por bloco & 0,02 & 0,10 & 0,10 & 0,14 & 0,09 & 0,10 & 0,06 & 0,11 \\
\hline Mudanças de TR por IIE & 0,12 & 0,10 & 0,07 & 0,10 & 0,05 & 0,06 & 0,04 & 0,07 \\
\hline EP das mudanças de TR por IIE & 0,29 & 0,21 & 0,18 & 0,26 & 0,09 & 0,19 & 0,11 & 0,25 \\
\hline
\end{tabular}

$\mathrm{CPT}=$ teste de desempenho contínuo; $\mathrm{DP}=$ desvio padrão; $\mathrm{EP}=$ erro padrão; IIE = intervalo interestímulo; TR = tempo de reação.

Tabela 4 - Comparação entre amostras de crianças brasileiras e norte-americanas para TR, erros de coomissão e omissão e perseverações

\begin{tabular}{|c|c|c|c|c|c|c|c|c|c|c|c|c|c|}
\hline \multirow{2}{*}{$\begin{array}{l}\text { Faixa etária/ } \\
\text { Gênero }\end{array}$} & \multirow[b]{2}{*}{ Amostra } & \multicolumn{2}{|c|}{$\begin{array}{c}\text { TR } \\
(\log )\end{array}$} & \multirow{2}{*}{$\begin{array}{c}\text { Estatística } \\
\text { (z) }\end{array}$} & \multicolumn{2}{|c|}{$\begin{array}{c}\% \\
\text { coomissão } \\
\end{array}$} & \multirow{2}{*}{$\begin{array}{c}\text { Estatística } \\
\text { (z) }\end{array}$} & \multicolumn{2}{|c|}{$\begin{array}{c}\% \\
\text { omissão } \\
\end{array}$} & \multirow{2}{*}{$\begin{array}{c}\text { Estatística } \\
\text { (z) }\end{array}$} & \multicolumn{2}{|c|}{ Preservações } & \multirow{2}{*}{$\begin{array}{c}\text { Estatística } \\
\text { (z) }\end{array}$} \\
\hline & & $\mathbf{M}$ & DP & & $\mathbf{M}$ & DP & & $\mathbf{M}$ & DP & & $\mathbf{M}$ & DP & \\
\hline \\
\hline \multirow[t]{2}{*}{ Masculino } & NA & 6,6 & 0,21 & $-76,40$ & 47,1 & 22,9 & 0,27 & 23,5 & 8,9 & $-6,15$ & 6,6 & 6,3 & $-2,01$ \\
\hline & Brasileira & & & & & & & & & $\mathrm{p}<0$ & & & $\mathrm{p}<0$, \\
\hline \multirow{2}{*}{ Feminino } & NA & 6,6 & 0,2 & 0,18 & 39 & 22 & $-1,41$ & 18 & 15 & $-1,66$ & & & $-2,28$ \\
\hline & Brasileira & 2,8 & 0,18 & $\mathrm{p}<0,001$ & 32,8 & 18,5 & $\mathrm{p}=0,079$ & 13,6 & 10,0 & $\mathrm{p}=0,048$ & & & $\mathrm{p}=0,011$ \\
\hline \multicolumn{14}{|c|}{100} \\
\hline \multirow[t]{2}{*}{ Masculino } & NA & 6,4 & 0,18 & $-108,19$ & 48,0 & 21,7 & $-1,96$ & 10,4 & 8,9 & 2,75 & 3,0 & 4,4 & $\begin{array}{l}-1, \\
=0\end{array}$ \\
\hline & $\begin{array}{c}\text { Brasileira } \\
\text { NA }\end{array}$ & $\begin{array}{l}2,8 \\
6,5\end{array}$ & $\begin{array}{l}0,07 \\
0,19\end{array}$ & $\begin{array}{c}\mathrm{p}<0,001 \\
-88,75\end{array}$ & $\begin{array}{l}40,0 \\
35,3\end{array}$ & $\begin{array}{l}18,7 \\
23,5\end{array}$ & $-0,38$ & $\begin{array}{l}5,8 \\
9,3\end{array}$ & $\begin{array}{l}5,7 \\
8,8\end{array}$ & $\begin{array}{c}\mathrm{p}=0,0030 \\
-1,81\end{array}$ & $\begin{array}{l}1 \\
2\end{array}$ & & $\begin{array}{c}\mathrm{p}=0,090 \\
-0,82\end{array}$ \\
\hline Feminino & Brasileira & 2,8 & 0,07 & $\mathrm{p}<0,001$ & 33,3 & 22,1 & $\mathrm{p}=0,349$ & 5,9 & 5,0 & $\mathrm{p}=0,0348$ & 1,6 & 1,8 & $\mathrm{p}=0,2051$ \\
\hline
\end{tabular}

$\mathrm{DP}=$ desvio padrão; $\mathrm{M}=$ média; $\mathrm{NA}=$ norte-americana; $\mathrm{TR}=$ tempo de reação

Tabela 5 - Comparação entre crianças brasileiras e norte-americanas para as outras medidas

\begin{tabular}{|c|c|c|c|c|c|c|c|c|c|c|c|c|c|}
\hline \multirow{2}{*}{$\begin{array}{l}\text { Faixa etária/ } \\
\text { Gênero }\end{array}$} & \multirow[b]{2}{*}{ Amostra } & \multicolumn{2}{|c|}{$\begin{array}{l}\text { Mudanças } \\
\text { de TR por } \\
\text { bloco }\end{array}$} & \multirow{2}{*}{$\begin{array}{c}\text { Estatística } \\
\text { (z) }\end{array}$} & \multicolumn{2}{|c|}{$\begin{array}{c}\text { EP das } \\
\text { Mudanças } \\
\text { de TR }\end{array}$} & \multirow{2}{*}{$\begin{array}{c}\text { Estatística } \\
\text { (z) }\end{array}$} & \multicolumn{2}{|c|}{$\begin{array}{l}\text { Mudanças } \\
\text { de TR } \\
\text { por IIE }\end{array}$} & \multirow{2}{*}{$\begin{array}{c}\text { Estatística } \\
\text { (z) }\end{array}$} & \multicolumn{2}{|c|}{$\begin{array}{c}\text { EP das } \\
\text { Mudanças de } \\
\text { TR po IIE }\end{array}$} & \multirow{2}{*}{$\begin{array}{c}\text { Estatística } \\
\text { (z) }\end{array}$} \\
\hline & & $\mathbf{M}$ & $\overline{\text { DP }}$ & & $\mathbf{M}$ & $\overline{\text { DP }}$ & & $\mathbf{M}$ & $\overline{\text { DP }}$ & & $\mathbf{M}$ & DP & \\
\hline \multicolumn{14}{|l|}{4} \\
\hline \multirow{2}{*}{ Masculino } & & $00 ?$ & 058 & & 0,11 & 0,13 & & & 0,13 & & 0,24 & 0,23 & \\
\hline & ras & & & & & & & & & & 0,2 & & \\
\hline \multirow{2}{*}{ Feminino } & & 0,0 & 0,06 & 0,02 & 0,08 & 0,13 & 0,7 & 0,1 & 0,1 & & 0,23 & 0,2 & $-1,07$ \\
\hline & Brasileira & 0,00 & 0,05 & 0,511 & 0,10 & 0,14 & $\mathrm{p}=0,7697$ & 0,07 & 0,10 & $\mathrm{p}=0,0139$ & 0,18 & 0,26 & $\mathrm{p}=0,140$ \\
\hline \multirow{2}{*}{5 Masculino } & & & & & & & & & & & & & \\
\hline & Brasi & & & 001 & & & $y=0$ & & & $p-0$ & 0,09 & & 30 \\
\hline \multirow{2}{*}{ Feminino } & & & & & & 0 , & & & & & 0,15 & 0,0 & \\
\hline & Brasileira & 0,00 & 0,05 & $\mathrm{p}=0,0100$ & 0,06 & 0,11 & $\mathrm{p}=0,0509$ & 0,04 & 0,07 & $\mathrm{p}=0,0600$ & 0,11 & 0,25 & $\mathrm{p}<0,238$ \\
\hline
\end{tabular}

$\mathrm{EP}=$ erro padrão; IIE = intervalo interestímulo; $\mathrm{M}=$ Média; $\mathrm{NA}=$ norte-americana; $\mathrm{TR}=$ tempo de reação. 
omissões, TR, EP do TR e mudanças de TR por bloco. Os meninos brasileiros de 5 anos tiveram melhores escores para porcentagem de coomissões e para EP das mudanças de TR por IIE, e as meninas brasileiras de 5 anos também tiveram taxas melhores na medida de EP das mudanças de TR por bloco.

\section{Discussão}

No presente estudo, foram observados efeitos de idade e gênero sobre o desempenho. Esses fatores foram altamente relevantes na determinação do desempenho em várias medidas do CPT em crianças mais velhas ${ }^{15,16}$. Deve-se lembrar, contudo, que há muito poucos estudos que investigaram esses efeitos e que as versões dos CPTs aplicadas em cada um deles são significativamente diferentes, dificultando comparações.

No que se refere à idade, encontramos um melhor desempenho em crianças mais velhas, o que está de acordo com estudos anteriores ${ }^{2,8,18}$. Especificamente, a idade foi importante para determinar a melhora no escore das medidas de variabilidade, perseverações, mudanças de TR por IIE e EP das mudanças de TR por IIE, conforme observado em crianças em idade escolar ${ }^{5,10}$.

As meninas brasileiras cometeram menos erros de coomissão, tiveram melhor capacidade para distinguir sinais (índice d') e foram mais cautelosas ao responder (índice $\beta$ ) do que os meninos brasileiros, confirmando achados anteriores em crianças mais velhas ${ }^{5,8}$. Além disso, os efeitos de gênero sobre as medidas d $\mathrm{d}^{5,15}, \beta$ e erros de coomissão $0^{2,10,15,16}$ são consistentes com achados anteriores em crianças mais velhas quando diferentes versões de CPT foram usadas.

Com relação às diferenças de gênero na atenção sustentada de crianças de 6 anos ou mais novas, Conners ${ }^{2}$, que forneceu as normas norte-americanas para o K-CPT, não analisou tais efeitos. Kerns \& Rondeau ${ }^{8}$, que analisaram esses efeitos, não observaram diferenças de gênero no desempenho, o que está em desacordo com nossos achados, possivelmente porque esses autores utilizaram uma versão diferente do teste (teste C-CPT) e seus sujeitos tinham um variação de idade maior (3 a 6 anos), o que poderia ter mascarado os efeitos observados aqui.

Em uma comparação entre as amostras brasileira e norte-americana, detectamos diferenças em várias medidas, de acordo com o que foi observado anteriormente para crianças mais velhas ${ }^{5}$. Os meninos e as meninas brasileiros tiveram escores melhores do que as crianças norte-americanas para TR (TR, EP do TR) e porcentagem de erros de omissão. Nas medidas restantes, as diferenças dependeram, ainda, da idade e do gênero, e novamente mostraram escores melhores para a amostra brasileira.

$\mathrm{O}$ achado de melhores escores das crianças brasileiras pode ser interpretado de duas formas considerando-se que as experiências socioculturais podem influenciar o funcionamento cognitivo, assim como o gênero, a idade e a escolaridade ${ }^{11}$, o que ainda é pouco conhecido ${ }^{19}$ : ou esse teste é sensível a tais fatores culturais ainda não especificados ou as diferenças se originam de diferenças na metodologia ${ }^{5}$. Com relação à primeira hipótese, poderia se argumentar que os efeitos de idade e gênero obtidos no presente estudo foram, de alguma forma, equivalentes àqueles previamente relatados em diferentes locais através da utilização de outras versões de CPT em crianças mais velhas, corroborando a idéia geralmente aceita de que o desempenho em medidas de CPT é relativamente constante em culturas diferentes ${ }^{11}$. No que se refere à segunda hipótese, devido ao fato da presente amostra e da amostra norte-americana terem sido comparadas por idade e escolaridade, os resultados poderiam somente ser explicados por diferenças de classe social, diferente proporção de meninas e meninos em cada faixa etária e/ou triagem para déficit de atenção. $\mathrm{O}$ autor do manual do K-CPT ${ }^{9}$ não informou o status socioeconômico dos sujeitos; portanto, pode ser que sua amostra tenha incluído somente crianças de classe social mais baixa do que as crianças da amostra brasileira, e, consequentemente, seus sujeitos tiveram escores piores. Isso seria surpreendente, porque, no presente estudo, os sujeitos foram selecionados de maneira a refletir a distribuição de classes sociais da cidade de São Paulo, localizada em um país menos desenvolvimento do que os EUA e no qual uma porcentagem muito maior de pessoas pertence a classes sociais mais baixas se comparado ao que ocorre na maioria das cidades norte-americanas. Com relação à distribuição de gênero, apesar da existência de uma proporção global equivalente entre meninos e meninas na amostra norte-americana, não foram fornecidas informações sobre a equivalência dessa proporção em cada faixa etária. Não acreditamos que possíveis diferenças nessa distribuição sejam uma explicação plausível para os nossos resultados, já que uma preponderância de meninos ou de meninas teria levado a efeitos de gênero diferentes daqueles previamente relatados.

Acreditamos que nossos achados referentes a escores globais melhores em comparação às normas norte-americanas sejam mais facilmente explicados pelas diferenças no procedimento de triagem ${ }^{5}$. Primeiramente, no presente estudo, a porcentagem de sujeitos para os quais se excluiu a possibilidade de distúrbios de desenvolvimento e/ou comportamentais é compatível com a literatura, apesar das variações entre os estudos ${ }^{20}$. Como as crianças com tais distúrbios têm um desempenho pior com relação à atenção sustentada ${ }^{7,18}$, se seus escores tivessem sido incluídos nas médias globais, o desempenho provavelmente teria sido pior e poderia ser compatível com os dados obtidos nos EUA. Na verdade, Conners ${ }^{9}$ reconhece que seus sujeitos não passaram por uma pré-triagem "portanto, uma proporção dos dados da população em geral incluiria casos com problemas de atenção" (p. 52) (tradução livre com base na versão do texto em inglês). A principal implicação disso é que os estudos normativos de CPT devem incluir triagem para distúrbios de atenção se seu propósito for o de serem usados para avaliar a probabilidade das crianças de terem déficits de atenção. Do contrário, um grande número de resultados falso-negativos pode ser obtido. 
Devido ao fato de "o padrão de desempenho poder ser inteiramente diferente em amostras clínicas"18 (p. 16) (tradução livre), a falta de triagem dificulta a determinação do efeito do contexto cultural sobre a atenção sustentada durante o desenvolvimento, assim como ocorre com o efeito de idade e de gênero. Além disso, a falta de triagem pode distorcer os escores em testes do tipo do K-CPT, possivelmente levando a problemas no diagnóstico do TDAH e de outros distúrbios de atenção nessa faixa etária.

Contudo, algumas limitações do nosso estudo devem ser apontadas. Primeiro, houve uma considerável perda de sujeitos da amostra original devido, principalmente, à falta de consentimento dos pais, de forma que o tamanho de nossa amostra final foi bastante reduzido. Além disso, avaliamos sujeitos de uma única cidade. Assim, para se obter normas brasileiras para o K-CPT, são necessários estudos futuros que incluam amostras maiores, envolvendo crianças de várias regiões do Brasil, assim como grupos clínicos para que parâmetros psicométricos adequados para a nossa população sejam determinados.

Apesar dessas limitações, nosso estudo é consistente com dados anteriores sobre efeitos de idade e de gênero e demonstra claramente que as normas norte-americanas não são adequadas para uso local. Portanto, nossos dados podem ser utilizados como um guia para possibilitar a definição do desvio dos escores esperados para idade e gênero que algumas crianças de 4 e 5 anos podem apresentar, tornando viável a identificação de possíveis distúrbios de atenção no Brasil.

\section{Conclusões}

Em suma, os procedimentos de triagem utilizados neste estudo, além do achado de efeitos de idade e de gênero previamente descritos sobre a atenção sustentada, indicam que os resultados descritos no presente estudo constituem escores de desempenho local apropriados e, como tal, são úteis na avaliação de crianças brasileiras em idade pré-escolar até que normas locais sejam estabelecidas.

\section{Agradecimentos}

Os autores gostariam de agradecer e expressar sua gratidão a Camila Cruz Rodrigues pela sua ajuda com a coleta de dados; às escolas EE Pandiá Calógeras, Externato N. Sra.
Menina, EE Heitor Carusi, EE Padre Manoel de Paiva, EE Homero dos Santos Fortes pela permissão e colaboração com a coleta de dados; e às crianças voluntárias e seus pais.

\section{Referências}

1. Riccio CA, Reynolds CR. Continuous performance tests are sensitive to ADHD in adults but lack specificity. A review and critique for differential diagnosis. Ann N Y Acad Sci. 2001;931:113-39.

2. Conners CK. Conners' continuous performance test for Windows [computer program]. Toronto: Multi-Health; 2002.

3. Epstein JN, Erkanli A, Conners CK, Klaric J, Costello JE, Angold A. Relations between Continuous Performance Test performance measures and ADHD behaviors. J Abnorm Child Psychol. 2003;31(5):543-54.

4. McGee RA, Clark SE, Symons DK. Does the Conner's Continuous Performance Test aid in ADHD diagnosis? J Abnorm Child Psychol. 2000;28(5):415-24.

5. Miranda MC, Sinnes EG, Pompeia S, Bueno OFA. A comparative study of performance in the Conner's Continuous Performance Test between Brazilian and North American children. J Atten Disord. 2008;11(5):588-98.

6. Smidts DP, Oosterlaan J. How common are symptoms of ADHD in typically developing preschoolers? A study on prevalence rates and prenatal/demographic risk factors. Cortex; 2007;43(6):710-7.

7. Mahone EM, Pillion JP, Hoffman J, Hiemenz JR, Denckla MB. Construct validity of the auditory continuous performance test for preschoolers. Dev Neuropsychol. 2005;27(1):11-33.

8. Kerns KA, Rondeau LA. Development of a continuous performance test for preschool children. J Atten Disord. 1998;2(4):229-38.

9. Conners CK. Conners' Kiddie continuous performance test for Windows [computer program]. Toronto: Multi-Health; 2000.

10. Conners CK, Epstein JN, Angold A, Klaric J. Continuous performance test performance in a normative epidemiological sample. J Abnorm Child Psychol. 2003;31(5):555-62.

11. Levav M, Mirsky AF, French LM, Bartko JJ. Multinational neuropsychological testing: performance of children and adults. J Clin Exp Neuropsychol. 1998;20(5):658-72.

12. Losier BJ, McGrath J, Klein RM. Error patterns on continuous performance test in non-medicated and medicated samples of children with and without ADHD: a meta-analytic review. J Child Psychol Psychiatry. 1996;37(8):971-87.

13. Brito GN. The Conners Abbreviated Teacher Rating Scale: development of norms in Brazil. J Abnorm Child Psychol. 1987;15(4):511-8.

14. Bussab WO, Morettin PA. Estatística básica. $4^{\text {a }}$ ed. São Paulo: Atual; 1987. Pp. 245-6.

15. Lin CC, Hsiao CK, Chen WJ. Developmental of sustained attention assessed using the continuous performance test among children $6-15$ years of age. J Abnorm Child Psychol. 1999;27(5):403-12.

16. Greenberg LM, Waldman ID. Developmental normative data on the test of variables of attention (T.O.V.A.). J Child Psychol Psychiatry. 1993;34(6):1019-30.

17. Denney CB, Rapport MD, Chung K. Interactions of task and subject variables among continuous performance tests. J Child Psychol Psychiatry. 2005;46(4):420-35.

18. Hagelthorn KM, Hiemenz JR, Pillion JP, Mahone EM. Age and task parameters in continuous performance tests for preschoolers. Percept Mot Skills. 2003;96(3 Pt 1):975-89.

19. Ratner C. Outline of a coherent, comprehensive concept of culture. Cross-Cult Psychol Bull. 2000;34(1-2):5-11.

20. Rohde LA, Halpern R. Transtorno de déficit de atenção/hiperatividade: atualização. J Pediatr (Rio J). 2004;80(2 supl):S61-70. 